\title{
Regulated Telescopic OTA Optimization for Mobile WiMAX Applications. Nano CMOS OTA Performance Prediction Through Bisquare Weights Method
}

\author{
Jihene Mallek, Houda Daoud, Hassene Mnif, and Mourad Loulou \\ Laboratory of Electronics and Information Technologies \\ University of Sfax, Tunisia \\ jihenemallek@yahoo.fr
}

\begin{abstract}
This paper presents the design of a high performance Regulated Telescopic Operational Transconductance Amplifier (OTA) for low power and high speed sigma-delta modulator for Mobile WiMAX Applications. Indeed, the Regulated Telescopic OTA is an enhanced DC gain Telescopic circuit with the gate voltage of the cascode transistor is controlled by a feedback amplifier. An algorithmic driven methodology is developed ending to the optimal transistor geometries. Moreover, the proposed OTA was post-layout simulated for some process corners, temperature variation and Monte-Carlo analysis. The post-layout simulation results have achieved a DC gain of $66 \mathrm{~dB}$, a large GBW of $862 \mathrm{MHz}$ with a phase margin of 58degrees with only $6.24 \mathrm{~mW}$ power consumption. In addition, the use of a robust Bisquare Weights (BW) method is investigated to predict the Regulated Telescopic OTA performance for new generation systems. Then, the impact of Nanometer CMOS on Regulated Telescopic OTA design is highlighted. It shows the potentialities of future CMOS processes to provide high speed and high performance OTA circuit.
\end{abstract}

Keywords: Regulated Telescopic OTA design; Heuristic optimization; DC gain; Bisquare Weights method; nanometer CMOS; predicted OTA performance

\section{Introduction}

The growing demand for high-speed and high-precision analog integrated circuits dictates stringent design specifications for the amplifiers which are the basic building blocks for numerous applications. In fact, high performance analog-to-digital converters (ADCs) require OTA circuit with very high DC gain and gain-bandwidth product (GBW), to meet both accuracy and fast settling requirements of the systems [1]. In addition, high-gain amplifiers use cascode structures or multi-stage designs with long channel length transistors biased at low current levels, while, high-bandwidth amplifiers use single-stage designs with short channel length transistors biased at high current levels [2]. Telescopic and folded-cascode structures are two common structures for single-stage OTAs. Two main drawbacks of the first one are low input common mode range and large voltage headroom in output. The main disadvantage of the folded one is that the power consumption is high and the GBW is low [3].

The OTA circuits can be achieved by exploiting the CMOS which is the preferred process for integrating systems on a chip. The CMOS process has been scaled according to Moore's Law, where the number of transistors on chips has grown at a faster rate in recent years [4]. Since process variations severely affect the characteristics of MOSFETs at the nanoscale, the prediction of analog circuit performances in sub-nm technologies is necessary to evaluate its capacities when designed using the future Nano-CMOS technology. In fact, performance estimation is a big challenge for analog circuit designers. Several approaches have been developed for efficient estimation. In fact, the least squares method is a standard method of adjustment which is elaborated by Legendre and Gauss [5]. It aims to get a smooth curve that fits the data points, $x 1 \ldots x i$ and it minimizes the sum of squared residuals [6]. The BW method is the most commonly used technique which is more accurate, yielding better predictions [7].

The objective of this work is to design a Regulated Telescopic OTA with a high DC gain

Received: May $18^{\text {th }}$ 2017. Accepted: December $18^{\text {th }}, 2019$

DOI: 10.15676/ijeei.2019.11.4.7 
and large GBW that satisfy the WiMAX application requirements. To achieve the targeted performance objectives, a Heuristic method used ending to the optimal OTA performance. Since the performance of this basic element circuit is closely related to the transistor scaling, we focus on evaluating the capacity of Regulated Telescopic OTA when designed using Nanometer CMOS process. We use the robust BW method to predict the Regulated Telescopic OTA performance during technology scaling.

The remaining of this paper is organized as follows. The design of the Regulated Telescopic OTA circuit is introduced in section 2. Analytical equations are investigated. The Heuristic method is presented in this section, together with optimized performance for the Regulated Telescopic OTA. Section 3 presents the post-layout simulation as well as process corners, temperature variation and Monte-Carlo analys is results. The predicted and optimal performance of the Regulated Telescopic OTA for future process is described in section 4. Finally, the main conclusions of this study are drawn in section 5.

\section{Regulated Telescopic OTA optimization}

Due to scaling of CMOS process technologies, lower supply range, and stronger focus on system-on-chip integration, analog design becomes more and more challenging. The operational transconductance amplifier, being an integral part of most analog systems, needs to address the stringent specifications on gain, bandwidth, linearity, noise and supply as well as stability [8]. The aim of this work is to design a Regulated Telescopic OTA instead of the conventional Telescopic OTA. In order to get more DC gain without changing the gainbandwidth product, Telescopic OTA with the gate voltage of the cascode transistor being controlled by a feedback amplifier [9]. The feedback is applied around the cascode transistor in order to enhance the gain. This feedback is in fact a parallel-series, causing the output impedance to increase with the amount of feedback gain. The gain rises with the same amount. So, the gain boosting adds another gain enhancement at low frequencies, without altering the GBW [10]. Figure 1 reveals the proposed Regulated Telescopic OTA. Despite, adding a feedback amplifier, the voltage swing of the Regulated Telescopic OTA at the output node was decreased and the layout area was raised, compared to the Telescopic OTA circuit.

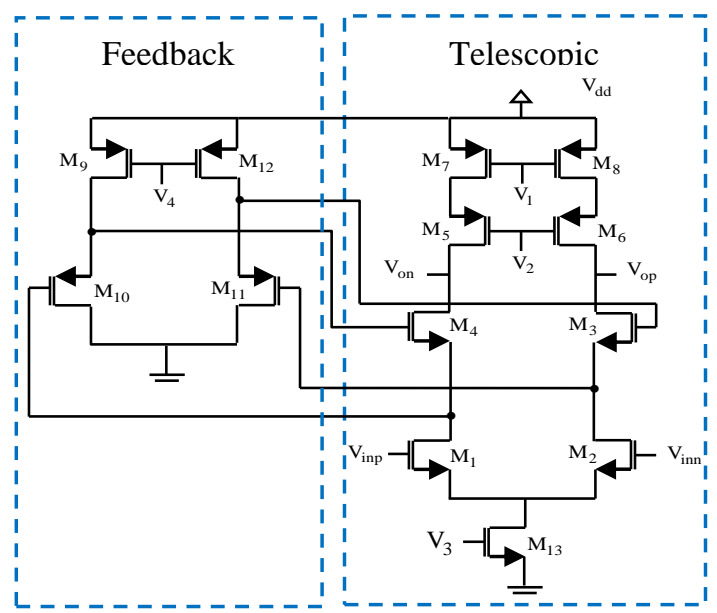

Figure 1. Schematic of the Regulated Telescopic OTA

Design of CMOS analog circuits are very significant in electronic system design. Day by day design complexity of electronic circuits is increased. So that, the optimization procedures should be automatic using greater precision. The key problems concern with electronic design automation are robustness and cost. Usually, optimization is a very exciting and timeconsuming task with many conflicting benchmarks and a wide range of design parameters. The proposed OTA can be designed using gm/ID methodology introduced by Flandre and Silveira 
[11]. However, we can only optimize the characteristics containing gm/ID parameter within expression's models [12].Thus, an alternative design approach is developed in this paper. It presents an optimization tool based on Heuristic algorithms [13]. We fixed our choice in this step of the AMS $0.35 \mu \mathrm{m}$ CMOS process. The Regulated Telescopic OTA optimization is based on maximizing the DC gain $\left(\mathrm{A}_{\mathrm{v}}\right)$, the GBW and the common mode rejection ratio (CMRR), and minimizing the input referred noise $\left(\mathrm{V}_{\mathrm{T}, \text { in }^{2}}{ }^{2}\right)$ and the silicon area of the whole OTA circuit. The objective function to maximize can thus be presented as follows:

$$
F_{0}=\theta_{1} A_{V}+\theta_{2} G B W+\theta_{2} C M R R+\frac{\theta_{4}}{V_{T_{1}{ }_{i n}^{2}}^{2}}+\frac{\theta_{5}}{\sum W_{i} L_{i}}
$$

Where $\theta_{1}, \ldots, \theta_{5}$ are positive coefficients used for normalization.

The first step in the optimization is the expression of the different criteria by a technology dependent model. For accurate modeling, a small signal analysis of the Regulated Telescopic OTA is carried out to explicit the different characteristics intended to optimize. From Regulated Telescopic OTA architecture presented in Figure1, the output resistance equation is given by the following equation:

$$
R_{\text {out }}=\left(\left(g m_{4}+g m_{10}\right) r o_{4}\left(\mathrm{ro}_{10} / / \mathrm{ro}_{9}\right) r o_{1}\right) / /\left(g m_{5} \mathrm{ro}_{5} \mathrm{ro}_{7}\right)
$$

Where $g_{m i}$ and $\mathrm{ro}_{\mathrm{i}}$ are respectively the transconductance of $\mathrm{M}_{\mathrm{i}}$ transistor for $\mathrm{i}=(4,5,10)$ and the drain-source resistance of $\mathrm{M}_{\mathrm{i}}$ device for $\mathrm{i}=(1,4,5,7,9,10)$. As a result, the open loop gain of the Regulated Telescopic OTA circuit is expressed as:

$$
A_{\mathrm{V}}=G_{m} R_{\text {out }}=g_{\mathrm{mi}}\left(\left(g m_{3}+g m_{10}\right) r o_{3}\left(\mathrm{ro}_{10} / / \mathrm{ro}_{9}\right) r o_{1}\right) / /\left(g m_{5} \mathrm{ro}_{5} \mathrm{ro}_{7}\right)
$$

The GBW is then given by:

$$
G B W=\frac{g_{m 1}}{2 \pi\left(C_{D B s}+c_{L}+C_{G D s}\right)}
$$

Where $\mathrm{C}_{\mathrm{DB} 3}$ and $\mathrm{C}_{\mathrm{GD} 3}$ are respectively the bulk drain capacitance and the drain gate capacitance of the $\mathrm{M}_{3}$ transistor and $\mathrm{C}_{\mathrm{L}}$ is the capacitance at the output node. The common mode rejection ratio (CMRR) can be approximated as:

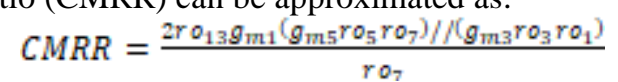

The input referred thermal noise voltage of the Regulated Telescopic OTA can be expressed as:

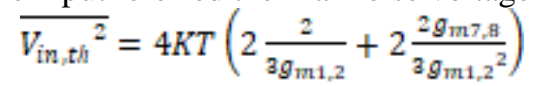

Where $\mathrm{K}$ is the Boltzmann's constant and $\mathrm{T}$ is the temperature. The input referred Flicker noise voltage of the Regulated Telescopic OTA can be written as:

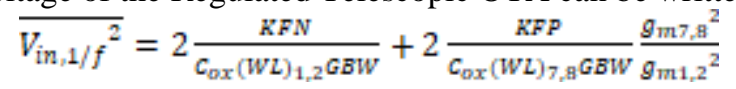

Where KFN and KFP are the Flicker noise coefficients of NMOS and PMOS transistors respectively. $\mathrm{C}_{\mathrm{ox}}$ is a constant for a given process. $\mathrm{W}$ and $\mathrm{L}$ represent the transistor sizes.

The heuristic and all constraints on each MOS transistor forming the Regulated Telescopic OTA were mathematically modelled, and developed in $\mathrm{C}++$ [14]. Thus, this program allowed us to teach high performance of OTA circuit. This approach was followed in several analog circuit designs and has produced promising results [15]. In fact, the heuristic algorithm used for optimizing the Regulated Telescopic OTA performances is essentially a random process. As shown in Figure 2, the mathematical models for both constraints and preliminary conditions to satisfy should be firstly developed. This program gives all possible parameters that are candidates for optimization taking into account their variations range. Secondly, the performance criteria and error sources are mathematically modeled. Afterwards, these models are taken into consideration in the program, and optimal parameters can be randomly selected from among the already calculated parameters. The objective function compilation to minimize or to maximize leads to optimal devices such as width and length values.

The optimization strategy which is essentially a random process, is detailed by the Figure 2 . Firstly, it initializes the parameters vector, which includes the size of the different MOS transistors interfering in the above analytical expressions. Next, the variable vector is selected and the preliminary requirements are checked. If these requirements are satisfied, then we can go the next step, otherwise, we make another choice. Afterward, we calculate the 
objective function. We record the parameters vector, if the objective function value has decreased. In fact, the testing vector is the parameters vector which includes the size of the different MOS transistors (width and length values) interfering in the analytical expressions. Moreover, at the end of heuristic algorithm, We record the new testing vector including the optimized size of the different MOS transistors.

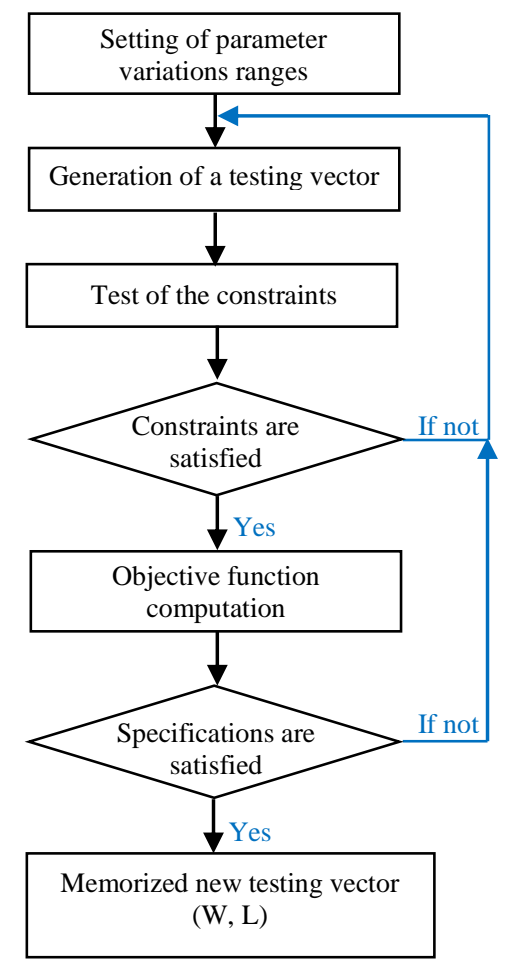

Figure 2. Flowchart of the heuristic

Simulation conditions are such as: the supply voltage is $3.3 \mathrm{~V}$ and the capacitor load is $1 \mathrm{pF}$. We notice that the optimization algorithm can be done in the same way for other simulation conditions. Table 1 summarizes the optimal device scaling that we get after applying the optimization algorithm. In addition, the designed Regulated telescopic OTA has a gain of $69.15 \mathrm{~dB}$ and a large GBW of $870 \mathrm{MHz}$ as presented in Figure 3. The transistor-level simulated performance is compared to theoretical design in Table 2. It is seen that the optimization procedure is almost satisfactory.

Table 1. Optimal device sizing

\begin{tabular}{|c|c|}
\hline Device name & Aspect ratio $(\mu \mathrm{m})$ \\
\hline $\mathrm{W}_{1,2,3,4}$ & $800 / 0.8$ \\
\hline $\mathrm{W}_{5,6,7,8}$ & $1100 / 0.5$ \\
\hline $\mathrm{W}_{13}$ & $590 / 0.5$ \\
\hline $\mathrm{W}_{9,12}$ & $900 / 0.8$ \\
\hline $\mathrm{W}_{10,11}$ & $200 / 0.8$ \\
\hline
\end{tabular}




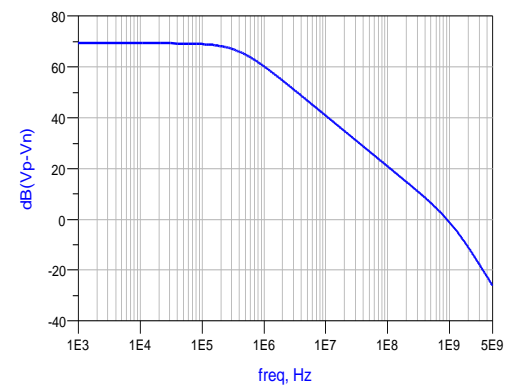

Figure 3. Gain curve

Table 2. Performance comparison

\begin{tabular}{|c|c|c|}
\hline Performance & Theoretical values & Simulated values \\
\hline DC gain $(\mathrm{dB})$ & 77.82 & 69.15 \\
\hline GBW $(\mathrm{MHz})$ & 970 & 870 \\
\hline CMRR $(\mathrm{dB})$ & 57.44 & 51 \\
\hline Slew rate $(\mathrm{V} / \mu \mathrm{s})$ & 395 & 351 \\
\hline Power consumption $(\mathrm{mW})$ & 6.34 & 6.22 \\
\hline
\end{tabular}

\section{Post-layout simulation results}

In order to evaluate the proposed Regulated Telescopic OTA design, various post-layout simulations are performed using AMS $0.35 \mu \mathrm{m}$ CMOS process parameters. The output frequency response of the Regulated Telescopic OTA, with $1 \mathrm{pF}$ load capacitance, is plotted in Figure 4. The Regulated Telescopic OTA has a DC gain of $66 \mathrm{~dB}$, a large GBW of $862 \mathrm{MHz}$ and a phase margin of 58 degrees. The Regulated Telescopic OTA performance is summarized in Table 3.

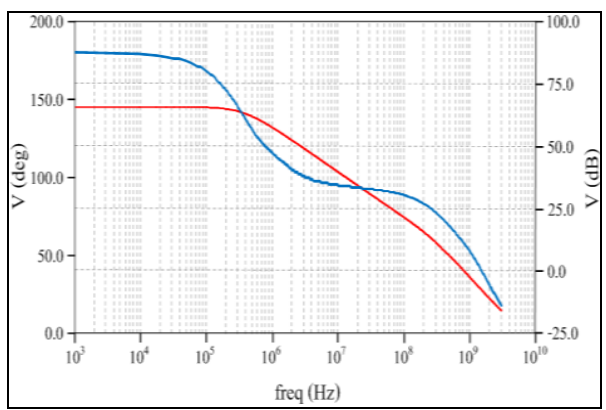

Figure 4. Gain and Phase curve

Table 3. Regulated telescopic OTA performance

\begin{tabular}{|c|c|c|}
\hline Performance & Transistor-level simulation & Post-layout simulation \\
\hline DC gain $(\mathrm{dB})$ & 69.15 & 66 \\
\hline GBW $(\mathrm{MHz})$ & 870 & 862 \\
\hline Phase margin $($ degrees) & 60 & 58 \\
\hline CMRR $(\mathrm{dB})$ & 51 & 49.4 \\
\hline Slew rate $(\mathrm{V} / \mu \mathrm{s})$ & \pm 351 & \pm 349 \\
\hline Settling time $(\mathrm{ns})$ & 14.24 & 14.3 \\
\hline Output-voltage swing $(\mathrm{V})$ & -1.5 to 1.5 & -1.2 to 1.2 \\
\hline Supply voltage $(\mathrm{V})$ & $0-3.3$ & $0-3.3$ \\
\hline Power consumption $(\mathrm{mW})$ & 6.22 & 6.24 \\
\hline Layout area $\left(\mu \mathrm{m}^{2}\right)$ & - & $(221 \times 202)$ \\
\hline
\end{tabular}


The Regulated Telescopic OTA is post-layout simulated for five process corners, namely: Fast Fast (FF), Typical Typical (TT), Fast Slow (FS), Slow Fast (SF) and Slow Slow (SS). Figure 5 shows the process corners post-layout simulation for gain curve. In addition, Table 4 lists the post-layout simulation performance of the Regulated Telescopic OTA for all the process corners. It can be clearly seen that the GBW, the Slew Rate, the settling time and the power consumption vary significantly over the process corners.

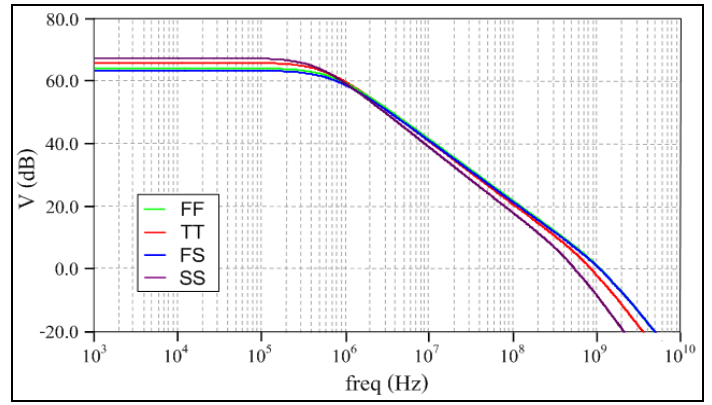

Figure 5. Process corners post-layout simulation for gain curve

Table 4. Post-layout simulation performance of the Regulated Telescopic OTA for all the process corners

\begin{tabular}{|c|c|c|c|c|c|}
\hline \multirow{2}{*}{ Performance } & \multicolumn{5}{|c|}{ Process Corners } \\
\hline & TT & $\mathrm{FF}$ & $\mathrm{FS}$ & SF & SS \\
\hline DC gain $(\mathrm{dB})$ & 66 & 64.02 & 63.22 & 67.32 & 67.18 \\
\hline GBW $(\mathrm{MHz})$ & 862 & 1090 & 1082 & 556 & 556 \\
\hline Phase margin (degrees) & 58 & 54.86 & 54.57 & 61.59 & 61.57 \\
\hline CMRR $(\mathrm{dB})$ & 49.4 & 48.31 & 47.96 & 50.48 & 50.19 \\
\hline Slew rate $(\mathrm{V} / \mu \mathrm{s})$ & \pm 349 & \pm 360 & \pm 357 & \pm 264.89 & \pm 263.84 \\
\hline Settling time (ns) & 14.3 & 13.85 & 13.97 & 17.74 & 17.78 \\
\hline Output-voltage swing (V) & -1.2 to 1.2 & -1.17 to 1.17 & -1.21 to 1.21 & -1.13 to 1.13 & -1.14 to 1.14 \\
\hline Power consumption $(\mathrm{mW})$ & 6.24 & 7.75 & 7.76 & 5.96 & 6.08 \\
\hline
\end{tabular}

The proposed OTA was post-layout simulated for temperature variation from $-20^{\circ} \mathrm{C}$ to $+100^{\circ} \mathrm{C}$. Figure 6 shows the temperature variations effect on the gain curve. Moreover, the post-layout Regulated Telescopic OTA performances for temperature variations are summarized in Table 5. It can be clearly seen that the GBW and the power consumption vary significantly across temperature variation.

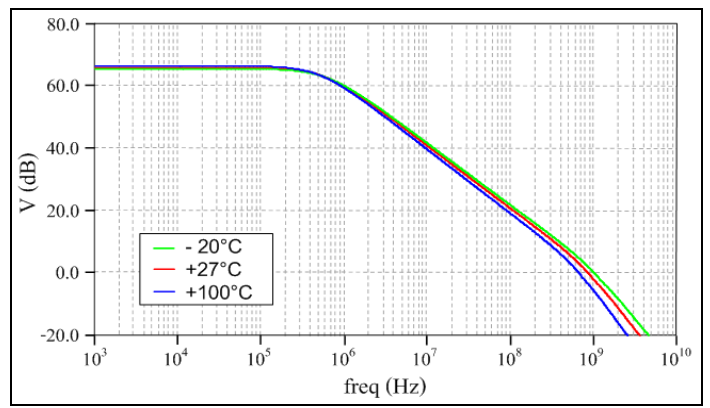

Figure 6. Gain curve with temperature variation from $-20^{\circ} \mathrm{C}$ to $+100^{\circ} \mathrm{C}$

Monte-Carlo simulations have been performed in order to check how the transistor mismatch affects performance of the Regulated Telescopic OTA circuit. Figure 7 shows the results of a Monte-Carlo simulation with 100 runs performed on the OTA circuit when all significant process parameters are varied by $\pm 10 \%$ from their nominal values. As can be seen from the histogram (Figure 7 ), $\pm 10 \%$ process parameters mismatches would result in an 
average DC gain of $63.54 \mathrm{~dB}$. Hence, the degradation is about $2.46 \mathrm{~dB}$. Furthermore, the main results of the Monte Carlo simulations are summarized in Table 6. It shows that the results of Monte-Carlo simulation have low variation from that of post-layout simulation results, except for the power consumption. We are focusing on the abilities evaluation of Regulated telescopic OTA when designed using future upcoming CMOS process. Moreover, we look forward to estimate the proposed OTA performance with process scaling.

Table 5. Regulated Telescopic OTA performance with temperature variation

\begin{tabular}{|c|c|c|c|}
\hline \multirow{2}{*}{ Performance } & \multicolumn{3}{|c|}{ Temperature } \\
\cline { 2 - 4 } & $-20^{\circ} \mathrm{C}$ & $+27^{\circ} \mathrm{C}$ & $+100^{\circ} \mathrm{C}$ \\
\hline DC gain $(\mathrm{dB})$ & 65.91 & 66 & 66.11 \\
\hline GBW $(\mathrm{MHz})$ & 1048 & 862 & 662 \\
\hline Phase margin (degrees) & 57 & 58 & 60.1 \\
\hline CMRR (dB) & 47.67 & 49.4 & 51.27 \\
\hline Slew rate $(\mathrm{V} / \mu \mathrm{s})$ & \pm 352 & \pm 349 & \pm 348 \\
\hline Settling time $(\mathrm{ns})$ & 14.28 & 14,3 & 14.31 \\
\hline Output-voltage swing $(\mathrm{V})$ & -1.21 to 1.21 & -1.2 to 1.2 & -1.13 to 1.13 \\
\hline Power consumption $(\mathrm{mW})$ & 5.45 & 6.24 & 6.75 \\
\hline
\end{tabular}

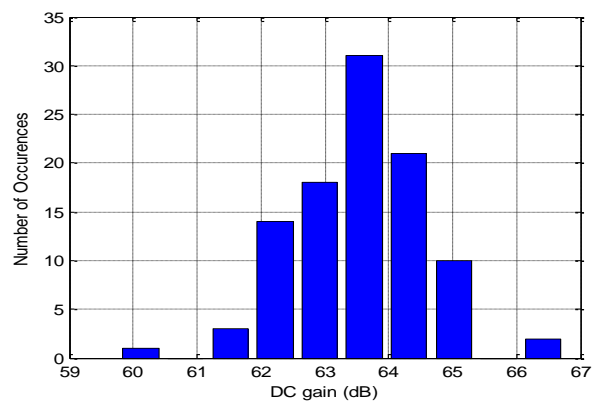

Figure 7. Histogram of 100 -run Monte-Carlo simulation showing the effect of $\pm 10 \%$ process parameters mismatches on DC gain

Table 6. Results of Monte-Carlo simulation

\begin{tabular}{|c|c|c|}
\hline Performance & Post-layout simulation & Monte-Carlo simulation \\
\hline DC gain $(\mathrm{dB})$ & 66 & 63.54 \\
\hline GBW $(\mathrm{MHz})$ & 862 & 897 \\
\hline Phase margin $($ degrees) & 58 & 59.24 \\
\hline CMRR (dB) & 49.4 & 47.44 \\
\hline Slew rate $(\mathrm{V} / \mu \mathrm{s})$ & \pm 349 & \pm 344 \\
\hline Settling time $(\mathrm{ns})$ & 14.3 & 14.35 \\
\hline Output-voltage swing $(\mathrm{V})$ & -1.2 to 1.2 & -1.12 to 1.12 \\
\hline Power consumption $(\mathrm{mW})$ & 6.24 & 7.44 \\
\hline
\end{tabular}

\section{Prediction of the Nano CMOS Regulated Telescopic OTA performance}

The aim of analog designers is to minimize the OTA power consumption through Nanometer CMOS processes. We place the accentuation on the prediction of Regulated Telescopic OTA performance using future CMOS processes. As shown in Figure 8, we begin by specifying the process node. Furthermore, we choose the parameters listed in Table7 including supply voltage $\left(\mathrm{V}_{\mathrm{dd}}\right)$, threshold voltage $\left(\mathrm{V}_{\mathrm{th}}\right)$, equivalent electrical oxide thickness $\left(\mathrm{T}_{\mathrm{oxe}}\right)$, channel doping concentration $\left(\mathrm{N}_{\mathrm{ch}}\right)$ etc... that are useful for OTA circuit design [6]. The analytical expressions that characterize the OTA circuit and the Nanoscale technological parameters are then used to apply the optimization process. Finally, the performance of 
Regulated Telescopic OTA such as DC gain, GBW, CMRR, Slew rate and power consumption are predicted and optimized.

Table 7. List of Nanoscale technological parameters

\begin{tabular}{|c|c|c|c|c|c|c|c|c|c|c|}
\hline $\begin{array}{c}\text { Process nodes } \\
(\mathrm{nm})\end{array}$ & \multicolumn{2}{|c|}{65} & \multicolumn{2}{c|}{90} & \multicolumn{2}{c|}{130} & \multicolumn{2}{c|}{180} & \multicolumn{2}{c|}{250} \\
\hline Data sources & \multicolumn{2}{|c|}{ INTEL $^{04}$} & \multicolumn{2}{c|}{ TSMC $^{02}$} & \multicolumn{2}{c|}{ INTEL $^{01}$} & \multicolumn{2}{c|}{ TSMC $^{09}$} & \multicolumn{2}{c|}{ TSMC $^{09}$} \\
\hline Device Type & $\mathrm{N}$ & $\mathrm{P}$ & $\mathrm{N}$ & $\mathrm{P}$ & $\mathrm{N}$ & $\mathrm{P}$ & $\mathrm{N}$ & $\mathrm{P}$ & $\mathrm{N}$ & $\mathrm{P}$ \\
\hline $\mathrm{V}_{\text {dd }}(\mathrm{V})$ & \multicolumn{2}{|c|}{1.2} & \multicolumn{2}{|c|}{1.2} & \multicolumn{2}{|c|}{1.4} & \multicolumn{2}{|c|}{1.8} & \multicolumn{2}{|c|}{2.5} \\
\hline $\mathrm{V}_{\text {th }}(\mathrm{V})$ & 0.28 & -0.24 & 0.29 & -0.22 & 0.33 & -0.25 & 0.37 & -0.38 & 0.37 & -0.52 \\
\hline $\mathrm{T}_{\text {ox }}(\mathrm{nm})$ & 1.85 & 1.95 & 2.05 & 2.2 & 2.15 & 2.3 & 4.1 & 4.1 & 5.7 & 5.7 \\
\hline $\mathrm{N}_{\mathrm{ch}}\left(\mathrm{e}^{18} \mathrm{~cm}^{-3}\right)$ & 5 & 5 & 3.5 & 3.5 & 2.5 & 2.5 & 0.23 & 0.41 & 0.23 & 0.41 \\
\hline
\end{tabular}

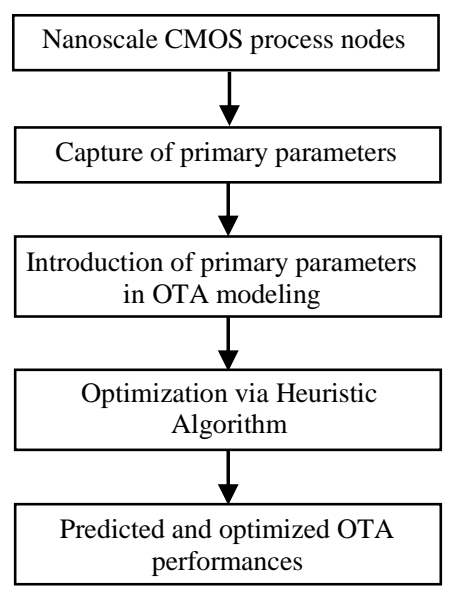

Figure 8. Synoptic of Nano CMOS OTA performance prediction

\section{A. Prediction method process}

The Bisquare Weights prodective method needs (xi, yi) scatter as long as a monotony combination of $\mathrm{x}$ and $\mathrm{y}$ parameters is set, in order to reach a smooth scaling [16, 17]. $\mathrm{x}$ and $\mathrm{y}$ parameters define respectively the process nodes and Regulated Telescopic OTA performance. Our object consists of fitting linear or non-linear models to data using the robust BW method from $45 \mathrm{~nm}$ to $22 \mathrm{~nm}$ process nodes.

Firstly, we use the Matlab command "cftool". We are often confronted with the task of determining the best model among various proposed alternatives, before fitting the scatter set (xi, yi). In order to choose the best fit, we compare different fit results, including the fitted coefficients and goodness of fit statistics. Moreover, the graphical and numerical fit results should be considered such as the residuals, the prediction bounds, the goodness of fit statistics and the confidence bounds. Afterwards, when the fit model is fixed, we extrapolate it to predict the Regulated Telescopic OTA performance from $45 \mathrm{~nm}$ to $22 \mathrm{~nm}$ process nodes. Finally, we plot both the predicted results and the data. We take as an example, residuals graphical display of different model fits as given in Figure 9. In fact, we fit (xi, yi) data with polynomial, exponential and power model fits. The residual for power model appears randomly scattered around zero proving that the model fit the data well. In addition, the polynomial and exponential models are not suitable fit for the data because the residuals are systematically negative for much of the data range. Besides, the numerical fit results are summarized in Table8. In fact, the power model represents the best fit since the associated SSE and RMSE values are the lowest.

To prove the robustness of BW method, we compared it with another method called leastsquare (LS) method. In fact, the LS method is a standard approach elaborated by Legendre and Gauss [5]. As shown in Figure 10, we are fitting the data with the power model using the LS 
and the BW methods. From this figure, it can be seen that the use of BW method results in a smooth and accurate fitting.

Table 8. Numerical fits results

\begin{tabular}{|c|c|c|c|c|c|}
\hline \multirow{2}{*}{ General model fit } & $\begin{array}{c}\text { Coefficients values } \\
\text { (with 95\% confidence bounds) }\end{array}$ & SSE & R-sq & Adj-R-sq & RMSE \\
\hline $\begin{array}{c}\text { Linear polynomial: } \\
\mathrm{f}(\mathrm{x})=\mathrm{ax}+\mathrm{b}\end{array}$ & $\begin{array}{c}\mathrm{a}=0.039[0.025,0.052] \\
\mathrm{b}=65.15[62.46,67.84]\end{array}$ & 5.25 & 0.94 & 0.92 & 1.14 \\
\hline Power: $\mathrm{f}(\mathrm{x})=\mathrm{ax}^{\mathrm{b}}$ & $\begin{array}{c}\mathrm{a}=44.98[43.77,46.18] \\
\mathrm{b}=0.093[0.088,0.098]\end{array}$ & 0.14 & 0.99 & 0.99 & 0.19 \\
\hline $\begin{array}{c}\text { Exponential: } \\
\mathrm{f}(\mathrm{x})=\mathrm{aexp}(\mathrm{bx})\end{array}$ & $\begin{array}{c}\mathrm{a}=65.54[62.78,68.29] \\
\mathrm{b}=0.00052[0.00033,0.00072]\end{array}$ & 6.4 & 0.92 & 0.91 & 1.26 \\
\hline
\end{tabular}
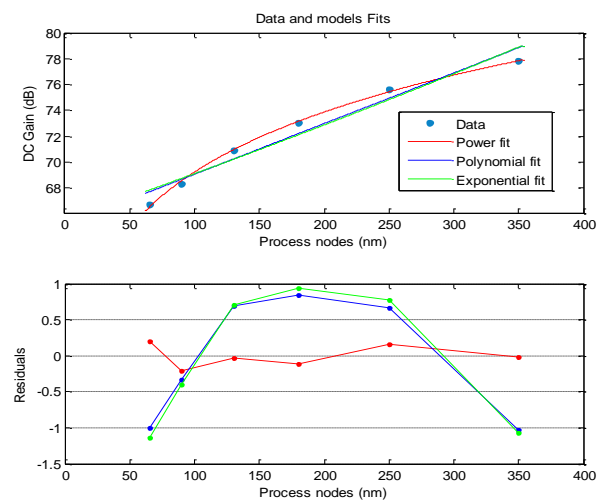

Figure 9. Graphical fits results

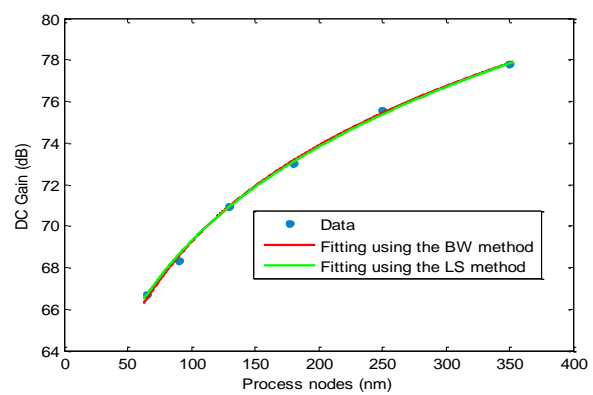

Figure 10. Fitting using both of the LS and the BW methods

\section{B. OTA performance prediction for Nano CMOS process}

High speed transistor can be provided, when analog designers move into a Nano CMOS process. The GBW varies between $0.97 \mathrm{GHz}$ and $6.61 \mathrm{GHz}$, it was noticed that the GBW of the Regulated Telescopic OTA was improved as shown in Figure 11. The GBW of upcoming transistor is continuously growing up with length decreasing of the transistor channel. The intrinsic gain of a transistor was decreased, hence, Figure 12 and Figure 13 prove the decline of Regulated Telescopic OTA DC gain and CMRR respectively. A gain-speed trade-off must be made in Nanometer CMOS process when faster speed and less gain are got from a single transistor. The Slew rate was decreased as shown in Figure 14, due to the strong rise of transconductance during the scaling process. The device characteristics become more sensitive to variations in the reduced channel length, making the OTA design tasks delicate. The shrinking of dimensions and supply voltage of the Regulated telescopic OTA circuit reduce significantly the power consumption. Figure 15 reveals the possible decrease of Regulated Telescopic OTA power consumption from $6.34 \mathrm{~mW}$ to $1.75 \mathrm{~mW}$. During Nanoscale process, in 
Jihene Mallek, et al.

spite of the reduction of DC gain, Regulated Telescopic OTA circuit presents acceptable DC gain useful for high speed applications.

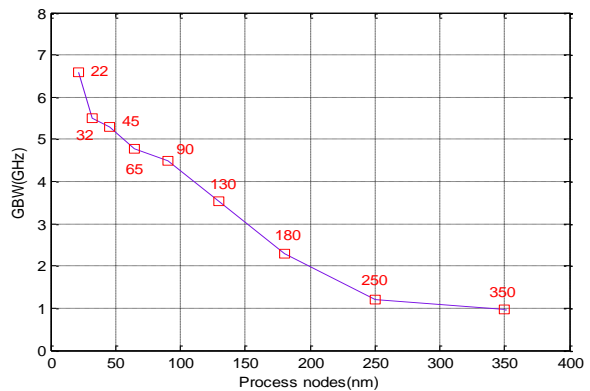

Figure 11. The scale of GBW

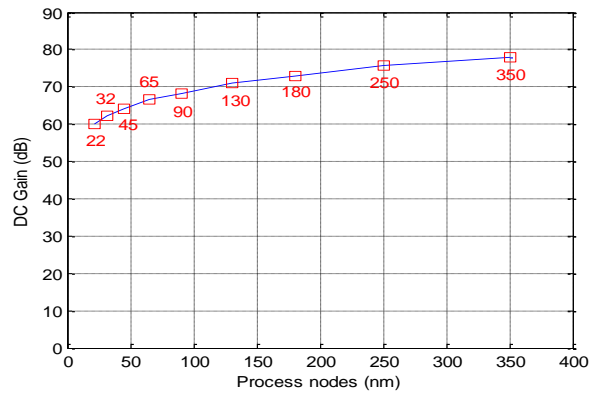

Figure 12. The scale of DC Gain

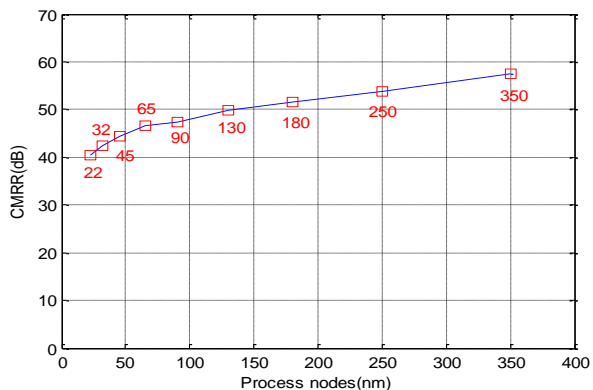

Figure 13. The scale of CMRR

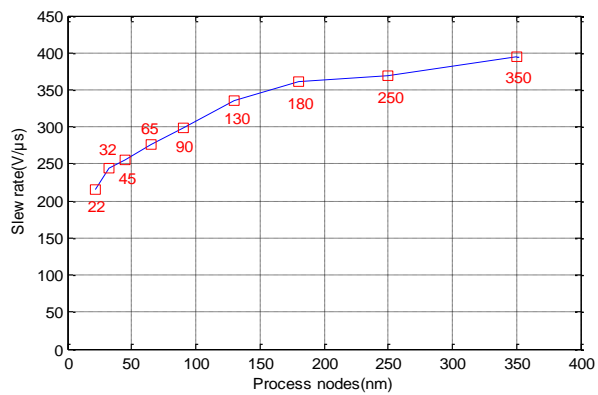

Figure 14. The scale of Slew rate 


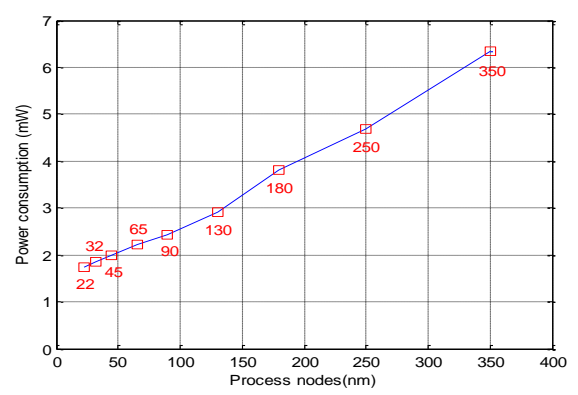

Figure 15. The scale of power consumption

\section{Evaluation of bisquare weights method}

In order to approve the robustness of the bisquare weights method, we interpolate the Nano CMOS Regulated Telescopic OTA Performances for $180 \mathrm{~nm}$ process nodes. In addition, we focus on comparing the interpolated OTA Performances with transistor-level simulation of the OTA circuit. The output frequency response of the Regulated Telescopic OTA for 180nm process nodes is plotted in Figure 16. The Regulated Telescopic OTA has a DC gain of $67.3 \mathrm{~dB}$, a GBW of $1.99 \mathrm{MHz}$. Table 9 summarizes the verification of $180 \mathrm{~nm}$ Bisquare Weights method with OTA transistor-level simulation. Moreover, the relative error given by Table 9 can be written as:

$$
\text { Relative error }=\frac{\text { real value }- \text { measured value }}{\text { real value }}
$$

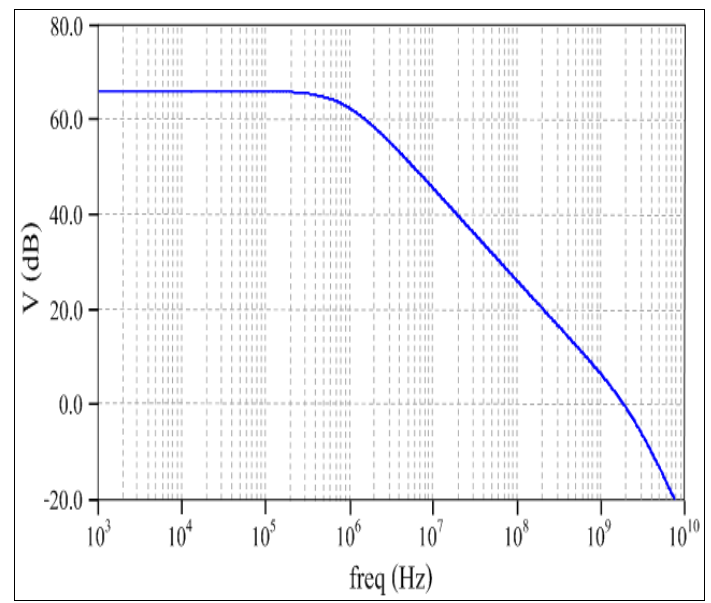

Figure 18. Gain curve for 180nm process nodes

Table 9. Verification of 180nm Bisquare Weights method with OTA transistor-level simulation

\begin{tabular}{|c|c|c|c|}
\hline & BW method & transistor-level simulation & Relative error $(\%)$ \\
\hline DC gain $(\mathrm{dB})$ & 73.14 & 65.90 & 10.9 \\
\hline GBW $(\mathrm{MHz})$ & 2.18 & 1.99 & 9.5 \\
\hline CMRR $(\mathrm{dB})$ & 51 & 46.43 & 9.8 \\
\hline Slew rate $(\mathrm{V} / \mu \mathrm{s})$ & 360 & 330 & 9 \\
\hline Power consumption $(\mathrm{mW})$ & 3.69 & 3.65 & 1 \\
\hline
\end{tabular}

The real value and the measured value represent, respectively the values given by OTA transistor-level simulation and BW methods. We conclude that the values of Regulated Telescopic OTA Performance interpolated with the BW method is approximately close to those given by the transistor-level simulation with a deviation of less than $11 \%$. Therefore, a 
satisfactory agreement between interpolated and transistor-level simulation of OTA performance is achieved. Then, the robust Bisquare Weights method proves the physicality and scalability of the performed interpolation. Otherwise, we verified the effectiveness of Bisquare Weights method by extrapolation. In fact, the values of Regulated telescopic OTA performance, predicted by BW method from $45 \mathrm{~nm}$ to $22 \mathrm{~nm}$ process nodes, have been carried out. Table 10 summarizes the estimated performance of the proposed OTA in comparison with other OTAs circuit presented recently. Relying on this comparison table, the values of OTA performance predicted with BW method are approximately close to those given by the literature. Therefore, the BW method proves the scalability of its extrapolation.

Table 10. Comparisons of estimated OTA Performance with other literatures

\begin{tabular}{|c|c|c|c|c|c|c|}
\hline Performance & \multicolumn{3}{|c|}{ This work } & {$[18]$} & {$[19]$} & {$[20]$} \\
\hline Process (nm) & 45 & 32 & 22 & 32 & 45 & 50 \\
\hline Supply Voltage (V) & 1 & 0.96 & 0.92 & 1 & 1.3 & 1 \\
\hline DC gain $(\mathrm{dB})$ & 64.2 & 62.16 & 55.9 & 53.43 & 57.83 & 57.15 \\
\hline GBW $(\mathrm{GHz})$ & 5.3 & 5.5 & 6.6 & 0.741 & 0.074 & 0.604 \\
\hline CMRR (dB) & 44.29 & 42.32 & 40.36 & - & - & - \\
\hline Slew rate $(\mathrm{V} / \mu \mathrm{s})$ & 255 & 245 & 215 & - & - & - \\
\hline Power consumption $(\mathrm{mW})$ & 2 & 1.85 & 1.75 & - & 0.042 & 0.56 \\
\hline
\end{tabular}

\section{Conclusion}

In this work, the design and implementation of a Regulated Telescopic OTA intended for use in Sigma-Delta modulator for Mobile WiMAX Applications were achieved. In fact, our design technique aimed at keeping an enhanced DC gain Telescopic OTA. For this reason, the telescopic OTA circuit with the gate voltage of the cascode transistor is controlled by a feedback amplifier. Moreover, an efficient optimization tool based on Heuristic algorithms was developed ending to the optimal transistor geometries. Behavioral transistor-level and postlayout simulations were presented. Then, we investigated both the process corners and the temperature variation as well as the Monte-Carlo analysis for Regulated Telescopic OTA to provide a better prediction for the experimental performance. The post-layout simulation results achieve high open-loop DC gain of $66 \mathrm{~dB}$ and large measured GBW of $862 \mathrm{MHz}$. The power consumption is approximately equal to $6.24 \mathrm{~mW}$ under $3.3 \mathrm{~V}$ supply voltage. In addition, we focused on the use of the Bisquare Weights method to predict the Regulated Telescopic OTA performances using upcoming CMOS Nanoprocess. The Heuristic program plays a key role in optimizing the predicted performance of Regulated Telescopic OTA. The CMOS scaling down leads to not only make high performance OTA, but also overcome several implementation difficulties. Future works will involve the use of the BW method to predict the Sigma-Delta modulator performance for ADCs used in Mobile WiMAX receivers.

\section{References}

[1] A. Dendouga, N.E. Bouguechal, S. Kouda, and S. Barra, "Modeling of a second Order Sigma-Delta Modulator with Imperfections," International Journal on Electrical Engineering and Informatics, vol. 3, no. 2, 2011.

[2] R. Assaad, and J. S. Martinez, "Recent Advances on the Design of High-Gain Wideband Operational Transconductance Amplifiers," Journal of VLSI Design, 2009.

[3] T. Yang, J. Lu, N. Poore, J. Holleman, "A Current-Reuse Complementary-Input ChopperStabilized Amplifier for Neural Recording," IEEE International New Circuits and Systems Conference (NEWCAS), 2014.

[4] G. E. Moore, "No exponential is forever: but "Forever" can be delayed!," IEEE International Solid-State Circuits Conference, pp. 20-23, 2003.

[5] A. Björck, "Numerical Methods for Least Squares Problems," North Holland, Society for Industrial and Applied Mathematics, 1996. 
[6] H. Daoud, S. Benselem and M. Loulou, "NanoCMOS Folded Cascode OTA Performances Prediction Through Least-Square Method," IEEE International New Circuits and Systems Conference, 2011.

[7] H. Daoud, S. Bensalem, S. Lahiani, C. Bensalem, and M. Loulou, "NanoCMOS optimized DVCC-based quadrature voltage controlled oscillator performances prediction through bisquare-weights method," Analog Integrated Circuits and Signal Processing, Springer, 2019.

[8] S. A. Aamir, and J. J. Wikner, "A 1.2-V pseudo-differential OTA with commonmodefeedforward in 65-nm CMOS," IEEE International Conference on Electronics, Circuits, and Systems (ICECS), 2010.

[9] J. Mallek, H. Mnif, H. Daoud, and M. Loulou, "A fully-differential Regulated Telescopic Operational Transconductance Amplifier," International Conference on Circuits, Systems, Signal Processing, Communications and Computers, 2014.

[10] Karakuzulu, "Design of a Low Current Leakage Integrator for Non-Coherent Ultra-Wide Band Receiver," Master, Royal Institute of Technology, Sweden, 2012.

[11] F. Silveira, D. Flandre, and P.G.A. Jespers, "A gm/ID based methodology for the design of CMOS analog circuits and application to the synthesis of a SOI micropower OTA," IEEE Journal of Solid State Circuits, vol. 31, no. 9, september 1996.

[12] H. Daoud, S. Zouari, and M. Loulou, "Design of Fast OTAs in Different MOS Operating modes using $0.35 \mu \mathrm{m}$ CMOS Process," International conference on microelectronics ICM, December 2006.

[13] K. Nagaraju, S. Sivanagaraju, T. Ramana, and V. Ganesh, "Heuristic Approach for Distribution Systems Feeder Reconfiguration to Line Maximum Loadability," International Journal on Electrical Engineering and Informatics, vol. 4, no. 1, 2012.

[14] M. Fakhfakh, M. Loulou and N. Masmoudi, "An improved algorithm-driven methodology to optimize switched current memory cells by transistor sizing," IEEE International Conference on Electrical, Electronic and Computer Engineering, 2004.

[15] M. Fakhfakh, M. Loulou, and N. Masmoudi, "Optimizing Performances of Switched Current Memory Cells through a Heuristic," Journal of Analog Integrated Circuits ad Signal Processing, Springer, vol.50, pp. 115-126, 2006.

[16] Garcia, "Robust smoothing of gridded data in one and higher dimensions with missing values," Computational Statistics and Data Analysis, Elsevier, pp. 1167-1178, 2010.

[17] H. Daoud, S. BenSalem, S. Zouari, and M. Loulou, "Use of Robust Method for NanoCMOS Process Prediction Application to Basic Block Analog Circuit Design," Journal of Circuits, Systems, and Computers, vol. 21, 2011.

[18] S. More, "Aging Degradation and Countermeasures in Deep-submicrometer Analog and Mixed Signal Integrated Circuits," Thesis, University of Munich, 2012.

[19] S. Kumar, P. Jay, and R. Prasad, "Gain Improvement of Two Stage OPAMP Through Body Bias in 45nm CMOS Technology," International Journal of Research in Engineering and Technology, vol.03, pp. 945-948, 2014.

[20] H. Trang, P. X. Hoang, and T. H. Quan, "A Novel design of low-power, high speed OTA in 50nm-CMOS technology," The 12th IEEE International Symposium on Signal Processing and Information Technology (ISSPIT), Ho Chi Minh City-Vietnam, 2012. 


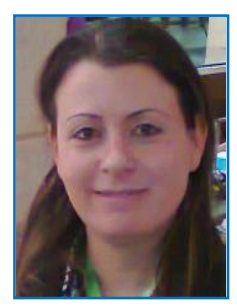

Jihene Mallek was born in Sfax, Tunisia, on September 1980. She received the Electrical Engineering Diploma then the Master degree in electronics from the National School of Engineering of Sfax, respectively, in 2006 and 2007. She joints the Electronic and Information Technology Laboratory of Sfax since 2006 and he has been a PhD student at the National School of Engineering of Sfax from 2006. She received her Ph.D. degree in 2017 in electronics from the same institution. His current research interests are on continuous time sigma delta ADC.

E-mail: jihenemallek@yahoo.fr

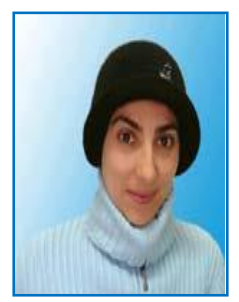

Houda Daoud Dammak was born in Sfax, Tunisia in 1980. She received the Electrical Engineering Diploma then the Master degree in electronics from the National School of Engineering of Sfax "ENIS", respectively, in 2004 and 2005. She received her Ph.D. degree in 2012 in electronics from the same institution. She joints the Electronic and Information Technology Laboratory of Sfax "LETI" since 2004 and she has been Assistant Professor at the National School of Electronic and Telecommunication of Sfax "ENET'com" from 2012. Her current research interests are on analogue CMOS integrated circuits design.

E-mail: daoud.houda@ieee.org

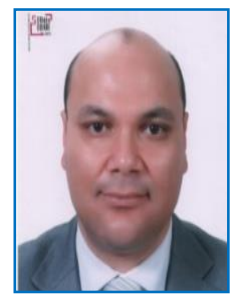

Hassene Mnif was born in Sfax, Tunisia, in 1975. He received the Dip Ing and Master in electrical engineering from the University of Sfax (ENIS) in 1999 and 2000, respectively, the $\mathrm{Ph}$. D. degree in electronics from the University of Bordeaux I, France, in 2004 and the HDR degree from the University of Sfax in 2011. He is currently Associate Professor and Director of studies in National School of Electronics and Telecommunications of Sfax, University of Sfax, where he has multiple innovative engineering education initiatives. He coordinates currently in Tunisia the European TEMPUS project "eSience". He is a member of Electronic Communication Group in the Electronic and Information Technology Laboratory. His research interests include characterization and compact modeling of both high frequency devices and future emerging technologies like Carbon Nanotube Field Effect Transistor (CNTFET). He participates also in researches in radiofrequency integrated and low power circuits design. He has authored and coauthored more than 60 journal publications and conference papers and has gathered significant scientific coordination experience within national and international collaborative research projects. He participated in the organization of several IEEE conferences and workshops, in particular ICECS 2009 and MELECON 2012.

E-mail: hassene.mnif@ieee.org

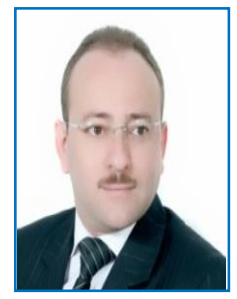

Mourad Loulou was born in Sfax, Tunisia in 1968. He received the Engineering Diploma from the University of Sfax in 1993 and his Ph.D. degree in 1998 in electronics from the University of Bordeaux France. Since October 2009 he became a Full Professor and he currently supervises the Analogue and RF IC Design Group at the National School of Engineers in Sfax. He is also a consulting expert in IC design. $\mathrm{He}$ is IEEE senior member. He is the organizer of the IEEE Tunisia Section and section chair from 2008 to 2012 . He is also the organizer and he chaired the Tunisia section Circuits And Systems Society chapter from 2009 to 2016. He served as general co-chair, TPC co-chair and committee member of several IEEE conferences. Professor Mourad Loulou, is advisor of several Ph. D. thesis in the field of the design and the design automation of Analogue, mixed mode and RF circuits. He is author and co-author of several journal papers, conference papers, book chapters and books.

E-mail: mourad.loulou@ieee.org 